OPEN ACCESS

Damping effects on centroid energies of isoscalar compression modes

To cite this article: D. C. Fuls et al 2010 EPL 9020006

View the article online for updates and enhancements.
Related content

Hot nuclei

S Shlomo and V M Kolomietz

- Exotic modes of excitation in atomic nuclei far from stability

Nils Paar, Dario Vretenar, Elias Khan et al.

Damping of small-amplitude nuclear

collective motion

J Wambach 


\title{
Damping effects on centroid energies of isoscalar compression modes
}

\author{
D. C. Fuls ${ }^{1}$, V. M. Kolomietz ${ }^{2}$, S. V. Lukyanov ${ }^{2}$ and S. Shlomo ${ }^{1(a)}$ \\ ${ }^{1}$ Cyclotron Institute, Texas A\&M University - College Station, TX 77643, USA \\ ${ }^{2}$ Institute for Nuclear Research - 03680 Kiev, Ukraine
}

received 4 January 2010; accepted in final form 20 April 2010

published online 20 May 2010

PACS 05.45.-a - Nonlinear dynamics and chaos

PACS 24.30.Cz - Giant resonances

PACS 21.60.Jz - Nuclear Density Functional Theory and extensions (includes Hartree-Fock and random-phase approximations)

\begin{abstract}
We study the sensitivities of the centroid energy $E 0$ of the isoscalar giant-monopole resonance and the centroid energy $E 1$ of the isoscalar giant-dipole resonance to the effect of relaxation. We find that the energy ratio $E 1 / E 0$ grows with the relaxation time approaching the experimental value $(E 1 / E 0)_{\exp }=1.6 \pm 0.1$
\end{abstract}

open Caccess Copyright (c) EPLA, 2010

Experimental data for the isoscalar giant-monopole resonances (ISGMR) and the isoscalar giant-dipole resonances (ISGDR) indicates that the centroid energies $E 1$ of the ISGDR are significantly smaller than those obtained by the self-consistent Hartree-Fock(HF)-based random phase approximation (RPA) calculations with effective interactions, which reproduce the experimental values of centroid energies $E 0$ of the ISGMR. The experimental value $(E 1 / E 0)_{\exp }=1.6 \pm 0.1[1-5]$ exceeds the prediction of the liquid-drop (hydrodynamic) model [3-5] and lies below the theoretical results for the ratio $E 1 / E 0$ obtained in both the RPA and the scaling-like calculations [6-11]. From a macroscopic (fluid dynamic) point of view, the ISGDR is caused by a compression and dilatation of the nucleus along some preferential direction with an unchanged nuclear volume. In contrast to the monopole (breathing) mode, the lowest isoscalar dipole excitation corresponds to a spurious center-of-mass motion and the ISGDR appears as an overtone. It is well known that the isoscalar compression modes correspond to zero sound excitations having the sound velocity $c_{0} \approx v_{F} \approx \sqrt{3} c_{1}$, where $v_{F}$ is the Fermi velocity and $c_{1}$ is the first sound velocity. This strong renormalization of the sound velocity by factor $\approx \sqrt{3}$ arises due to the Fermi surface distortion effect (FSDE).

However, the enhancement in the energy of the ISGMR, the lowest breathing mode, is strongly suppressed by the effects of the Fermi surface distortion on the boundary condition, resulting in a value for the energy of the ISGMR

${ }^{(a)}$ E-mail: shlomo@comp.tamu.edu which is close to that of the liquid-drop model (LDM), i.e., first sound regime prediction. This is not the case for higher breathing modes such as the overtone, see refs. $[11,12]$. Since the ISGDR appears as the overtone to the spurious mode, one can expect that the energy of the ISGDR should be shifted to higher energies with respect to the LDM prediction because the Fermi surface distortion effect is not compensated for by the boundary conditions. On the other hand, the FSDE depends significantly on inter-particle collisions and disappears in the limit of short relaxation time (hydrodynamic limit). Thus, one can expect that the ratio $E 1 / E 0$ will decrease and approach the experimental value if the collisional damping is taken into account.

We aim in this work to study the effect of collisional damping on the isoscalar compression modes, and find that one can achieve an agreement with the experimental data for the ratio $E 1 / E 0$ and for the widths of ISGMR and ISGDR in the case of a short relaxation time.

We will use the semi-classical kinetic approach in $(\mathbf{r}, \mathbf{p})$ phase space (fluid dynamic approach (FDA)). This approach, in contrast to the quantum HF-based RPA, ignores the single-particle (shell) effects. However, the advantage is that the kinetic theory allows one to take into consideration the relaxation (damping) processes in a transparent way due to the collisional integral [13].

In general, the isoscalar particle density variation $\delta \rho$ with respect to the equilibrium bulk density $\rho_{0}$ is given by

$$
\delta \rho(\mathbf{r}, t)=\int \frac{g \mathrm{~d} \mathbf{p}}{(2 \pi \hbar)^{3}} \delta f(\mathbf{r}, \mathbf{p} ; t) .
$$


Here, $g=4$ is the spin-isospin degeneracy factor and $\delta f(\mathbf{r}, \mathbf{p} ; t) \equiv \delta f$ is the isoscalar deviation of the distribution function $f(\mathbf{r}, \mathbf{p} ; t)$ from the equilibrium one $f_{\text {eq }}(\mathbf{r}, \mathbf{p})$.

A small variation of the distribution function $\delta f$ in eq. (1) can be evaluated using the linearized kinetic equation. In the nuclear volume, where inhomogeneity of the particle density is small, the quasi-particle concept of the Landau Fermi-liquid theory [14] can be justified. To evaluate $\delta f$ we will apply the linearized Landau-Vlasov equation, augmented by a source term $\delta \mathrm{St}[f]$ for relaxation processes, in the form $[13,15,16]$

$\frac{\partial}{\partial t} \delta f+\mathbf{v} \cdot \nabla_{\mathbf{r}} \delta f-\nabla_{\mathbf{r}}\left(\delta U_{\text {self }}+U_{\text {ext }}\right) \cdot \nabla_{\mathbf{p}} f_{\text {eq }}=\delta \operatorname{St}[f]$,

where $\mathbf{v}=\mathbf{p} / m^{*}$ is the quasi-particle velocity and $m^{*}$ is the effective mass of the nucleon.

We point out that the left-hand side of the kinetic equation (2) can be derived by the Wigner transformation from the linearized time-dependent Hartree-Fock equation in the presence of the external field $U_{\text {ext }}$ [17]. The variation of the self-consistent mean field $\delta U_{\text {self }}$ in eq. (2) is then given by the Wigner transformation of the corresponding mean field. The self-consistent mean field $\delta U_{\text {self }}$ is related to the Skyrme or Landau effective interaction. Within the Landau Fermi-liquid theory the quantity $\delta U_{\text {self }}$ can be derived in terms of Landau's interaction amplitude $v_{\text {int }}\left(\mathbf{p}, \mathbf{p}^{\prime}\right)$, see refs. $[13,15,18]$. We will also assume that the external field $U_{\text {ext }}(t)$ is periodic in time:

$$
U_{\text {ext }}(t)=\lambda_{0} e^{-i \omega t} \hat{q}+\lambda_{0}^{*} e^{i \omega t} \hat{q}^{*},
$$

where $\lambda_{0}$ is the small amplitude and $\hat{q}$ is the one-body operator.

The right-hand side of eq. (2) represents the change of the distribution function due to relaxation. In this work we use the approximation of independent dissipation rates. Namely, for small amplitude eigenvibrations and small deviations of the momentum distribution from the Fermi sphere we assume

$$
\delta \mathrm{St}[f]=-\frac{\delta f}{\tau_{\text {eff }}},
$$

where

$$
\frac{1}{\tau_{\mathrm{eff}}}=\frac{1}{\tau_{2}}+\frac{1}{\tau_{1}}+\frac{1}{\tau_{\uparrow}} .
$$

Here, the term $1 / \tau_{2}$ is due to the two-body collisions on the distorted Fermi surface, $1 / \tau_{1}$ determines the change in the distribution function resulting from one-body relaxation on the moving nuclear surface and $1 / \tau_{\uparrow}$ takes into account the possibility of particle emission.

The collisional relaxation time $\tau_{2}$ is frequency dependent. The frequency dependence of $\tau_{2}$ is caused by the memory (non-Markovian) effect in the collision integral. It can be shown, see chapt. 8 of ref. [14], that the presence of a fast collective eigenmode changes the energy conservation factor in the collision integral $\delta \mathrm{St}[f]$ and leads to a frequency dependence of the collisional relaxation time $\tau_{2}$.
Following Landau's prescription [14] at zero temperature, we will assume

$$
\tau_{2}=\frac{\hbar \beta}{\left(\hbar \omega_{R} / 2 \pi\right)^{2}},
$$

where $\omega_{R}$ is the real part of the eigenfrequency of sound mode. The coefficient $\beta$ depends on the $N N$ scattering cross-sections. We will use the value of $\beta=4.6 \mathrm{MeV}$ which corresponds to the isotropic energy-independent $N N$ cross-sections $\sigma_{p p}=\sigma_{n n}=25 \mathrm{mb}$ and $\sigma_{p n}=\sigma_{n p}=50 \mathrm{mb}$, see refs. $[19,20]$.

We now comment on the one-body relaxation and the possibility of treatment of this as a source term in the kinetic equation. The origin of this relaxation is related to the fragmentation width of the collective states in quantum calculations like the RPA. In the quantum RPA calculations, the fragmentation width does not reflect motion of the system towards thermal equilibrium but indicates rather a redistribution of the particle-hole excitations in the vicinity of the collective state. In our kinetic approach, we will imitate the fragmentation width by the one-body relaxation on the moving nuclear surface [21]. Note also that it was shown in refs. [21-23] that, in the classical limit for the random phase approximation, the fragmentation width coincides with the width obtained from the one-body relaxation mechanism. We use one-body relaxation as an additional source term in the kinetic equation with the relaxation time (see refs. $[19,21,24]$ )

$$
\tau_{1}=\frac{2 R_{0}}{\bar{v}} \xi,
$$

where $R_{0}$ is a nuclear radius, $\bar{v}=3 v_{F} / 4$ and $\xi$ is a free numerical factor which depends on the excitation mode.

For heavy nuclei, the value of the emission width $\Gamma_{\uparrow} \sim$ $1 / \tau_{\uparrow}$ is quite small $[25,26]$ and we neglect the contribution of the particle emission to the total relaxation time $\tau_{\text {eff }}$ (see also the comment after fig. 3).

The kinetic equation (2) can be reduced to local equations of motion in $\mathbf{r}$-space. Evaluating the $\mathbf{p}$-moments to the kinetic equation (2), one can reduce the kinetic equation to the equation for the particle density eigenvibrations, $\delta \rho_{\mathrm{vol}}$, in the nuclear interior [16]. Assuming $U_{\text {ext }}=0$, one obtains

$$
\frac{\partial^{2}}{\partial t^{2}} \delta \rho_{\mathrm{vol}}-c_{0}^{2} \nabla^{2} \delta \rho_{\mathrm{vol}}-\gamma \nabla^{2} \frac{\partial}{\partial t} \delta \rho_{\mathrm{vol}}=0,
$$

where $c_{0}$ is the zero sound velocity and $\gamma$ is the friction coefficient:

$$
c_{0}^{2}=\frac{1}{9 m}\left(K+12 \mu_{F} / \rho_{0}\right), \quad \gamma=\frac{4 \eta_{F}}{3 \rho_{0} m} .
$$

Here $K$ is the nuclear matter incompressibility and both kinetic coefficients, $\mu_{F}$ and $\eta_{F}$, depend on an unknown eigenfrequency $\omega$ of the isoscalar sound mode (see ref. [16] for details),

$$
\begin{aligned}
& \mu_{F}=\frac{3}{2} \rho_{0} s^{2} \epsilon_{F}\left[1-\frac{1}{3 s^{2}}\left(1+F_{0}\right)\right], \\
& \eta_{F}=\frac{3 \rho_{0} s^{2} \epsilon_{F}}{2 \omega_{R}^{2} \tau_{\text {eff }}}\left[1-\frac{1}{3 s^{2}}\left(1+F_{0}\right)\right],
\end{aligned}
$$


where $\epsilon_{F}$ is the Fermi energy, $F_{0}$ is the Landau interaction parameter [14], $s=\omega_{R} / v_{F} q, v_{F}$ is the Fermi velocity and $q$ is the wave number for the longitudinal sound wave. In general, the dimensionless sound velocity $s$ is obtained as a solution to the Landau's dispersion equation [14] and depends on the multipolarity $l$ of the Fermi surface distortion. Note that the zero sound velocity $c_{0}$ depends on the relaxation time because of eq. (10). In the frequentcollision limit, the Fermi distortion effect disappears and one has $c_{0} \rightarrow c_{1}$, where $c_{1}=\sqrt{K / 9 m}$ is the first sound velocity.

For the compression modes, the full variation of the particle density $\delta \rho(\mathbf{r}, t)$ also includes the contribution from the variation of the nuclear radius $R(t)=R_{0}[1+$ $\left.\alpha_{s}(t) Y_{L 0}(\hat{r})\right]$. For a given multipolarity $L$, the particle density variation $\delta \rho_{L}(\mathbf{r}, t)$ reads

$$
\begin{aligned}
\delta \rho_{L}(\mathbf{r}, t)= & \alpha_{L}\left[\theta\left(R_{0}-r\right) j_{L}(q r)+\frac{1-a \delta_{L 1}}{q} \delta\left(R_{0}-r\right)\right. \\
& \left.\times j_{L}^{\prime}\left(q R_{0}\right)\right] \rho_{0} Y_{L 0}(\hat{\mathbf{r}}) \exp (i \mathbf{q} \cdot \mathbf{r}-i \omega t),
\end{aligned}
$$

where $R_{0}$ is the equilibrium nuclear radius and the parameter $a$ is determined by the translation invariance condition in the case of the isoscalar dipole compression mode and is given by [27]

$$
a=j_{1}(x) / x j_{1}^{\prime}(x), \quad x=q R_{0} .
$$

We point out that expression (11) indicates that the nuclear surface is shifted in time for $L=0$ as well as for $L=1$ since $a \neq 1$. Due to this fact the one-body relaxation on the moving nuclear surface and the corresponding fragmentation width occur in both above-mentioned cases, see below. Note also that the one-body relaxation for the compression modes is possible due to the diffuse reflections of nucleons on the free nuclear surface, see refs. [24,28].

Expression (11) is a solution to the homogeneous equation (8) if the following dispersion equation is satisfied:

$$
\omega^{2}-c_{0}^{2} q^{2}+i \omega \gamma q^{2}=0
$$

Assuming $\omega=\omega_{R}+i \omega_{I}$, where $\omega_{R}$ and $\omega_{I}$ are real, one has

$$
\omega_{R}^{2}=c_{0}^{2} q^{2}-\frac{1}{4} \gamma^{2} q^{4}, \quad \omega_{I}=-\frac{1}{2} \gamma q^{2} .
$$

The amplitude $\alpha_{s}(t)$ is related to the motion in the nuclear interior because of the boundary conditions. The macroscopic boundary conditions for the consistent solutions of both the continuity and the Euler equations, taken at the moving nuclear surface, are given by [29]

$$
\begin{aligned}
\left.v_{r}\right|_{r=R_{0}} & =R_{0} \dot{\alpha}_{s} Y_{L 0}(\hat{r}), \\
\left.\delta P_{r r}^{(\mu)}\right|_{r=R_{0}} & =\alpha_{s} \mathcal{P}_{s} Y_{L 0}(\hat{r})\left(1-\delta_{L 1}\right),
\end{aligned}
$$

where $\mathcal{P}_{s}=2 \sigma / R_{0}$ is the capillary pressure and $\sigma$ is the nuclear surface tension coefficient. On the l.h.s. of eqs. (15) and (16) one has the radial components of the velocity field, $v_{r}$, and the pressure tensor, $\delta P_{r r}^{(\mu)}$, which are determined in the nuclear volume.

The isoscalar compression dipole excitation occurs at $\mathcal{P}_{s}=0$, and eq. (16) gives the following secular equation for $q$ :

$$
\left[\frac{1}{9}\left(K-6 \frac{\mu_{F}}{\rho_{0}}\right) j_{1}(q r)-2 \frac{\mu_{F}}{\rho_{0}} j_{1}^{\prime \prime}(q r)\right]_{r=R_{0}}=0, \quad \text { for } \quad L=1 .
$$

In the case of the isoscalar monopole excitation, the additional contribution from the surface pressure $\mathcal{P}_{s}$ in eq. (16) has to be taken into account and the corresponding secular equation reads (see ref. [11])

$$
\left[q r j_{0}(q r)-\left(f_{\sigma}+f_{\mu}\right) j_{1}(q r)\right]_{r=R_{0}}=0, \quad \text { for } \quad L=0
$$

where

$$
f_{\sigma}=\frac{2 \sigma}{m c_{0}^{2} \rho_{0} R_{0}}, \quad f_{\mu}=\frac{4 \mu_{F}}{m c_{0}^{2} \rho_{0}} .
$$

Here $f_{\sigma}$ appears due to the surface tension contribution to the boundary condition (16) with the surface tension coefficient $\sigma$.

In the microscopic approach, the properties of giant resonances are calculated within the fully self-consistent HF-based RPA. In the Green's function method [30] one evaluates the RPA Green's function,

$$
G=G_{0}\left(1+V_{\mathrm{ph}} G_{0}\right)^{-1},
$$

where $V_{\mathrm{ph}}$ is the particle-hole $(\mathrm{p}-\mathrm{h})$ interaction and $G_{0}$ is the free $\mathrm{p}-\mathrm{h}$ Green's function.

For isoscalar electric modes the transition operator has the form

$$
\hat{F}_{L}=\sum \hat{f}(i), \quad \hat{f}=f(r) Y_{L 0}(i)
$$

Here $L=(0,1,2, \ldots)$ is the angular momentum transferred to the ground state in the excitation. The strength function $S(E)$ associated with the scattering operator $\hat{F}$ is given by

$$
\begin{aligned}
S(E)= & \sum_{n}|\langle 0|\hat{F}| n\rangle|^{2} \delta\left(E_{n}-E_{0}-E\right)= \\
& \frac{1}{\pi} \operatorname{Im}[\operatorname{Tr}(\hat{f} G \hat{f})],
\end{aligned}
$$

where the sum is over the excited states $|n\rangle$ with eigenenergies $E_{n}$ of the nucleus. For the isoscalar monopole we use $f(r)=r^{2}$ and for the isoscalar dipole we adopt the scattering operator $f(r)=r^{3}-(5 / 3)\left\langle r^{2}\right\rangle r$. We emphasize that our HF-based RPA calculations are fully selfconsistent (the spurious state mixing is negligible) [31]. The centroid energy of the GMR is obtained from

$$
E_{R}=\frac{m_{1}}{m_{0}}
$$




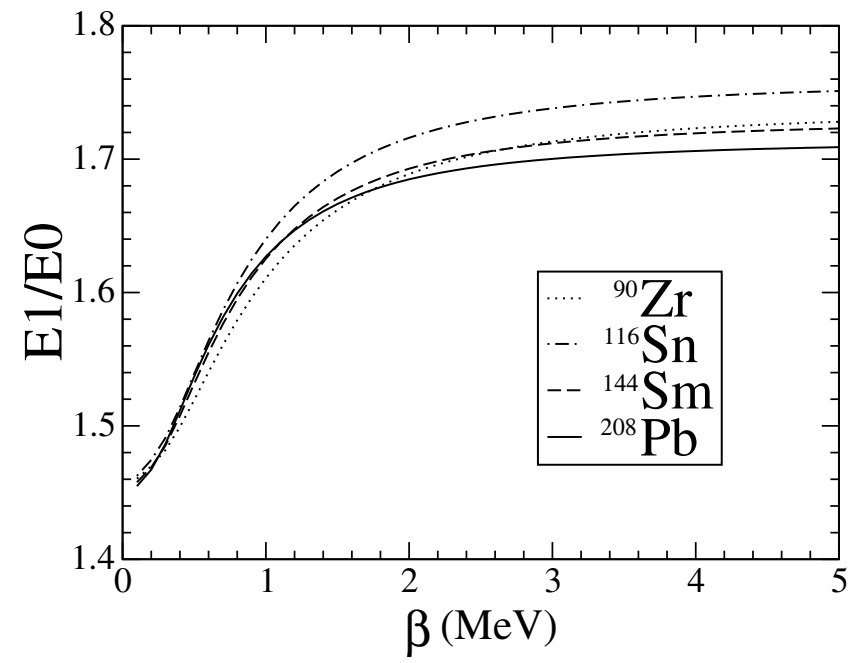

Fig. 1: Ratio of the energy $E 1$ of the isoscalar giant-dipole resonance to the energy $E 0$ of the isoscalar giant-monopole resonance for the nuclei ${ }^{90} \mathrm{Zr},{ }^{116} \mathrm{Sn},{ }^{144} \mathrm{Sm}$ and ${ }^{208} \mathrm{~Pb}$ as function of the damping parameter $\beta$ obtained within the fluid dynamic approach with $\tau_{1} \rightarrow \infty$.

where $m_{k}$ is the energy-weighted sum:

$$
m_{k}=\int_{E_{\min }}^{E_{\max }} E^{k} S(E) \mathrm{d} E .
$$

The range of integration $E_{\min }$ to $E_{\max }$ depends on the localization of the GMR.

We have evaluated the strength function $S(E)$ [31] within the self-consistent HF-based RPA using the Skyrme-type effective nucleon-nucleon interactions. We point out that, in contrast with the ISGMR, which contains a single peak, the dipole strength function displays a low-lying part which lies below the ISGDR. We have taken into account this fact using the range of integration $E_{\min }$ to $E_{\max }$ in $(24)$ which includes the energy distribution of the ISGDR (i.e., high-lying part of the dipole strength function) only. Note that this is consistent with our consideration of the ISGDR within the FDA.

The energies of the ISGMR and ISGDR can be evaluated using the dispersion relation (13) augmented by the secular equations (17) and (18). We stress that an important ingredient of our calculations is that the secular equations (17) and (18) for $q$ are solved simultaneously and consistently with the dispersion equation (13) for $s$ because $s$ also depends on $q$. We have carried out numerical calculations within the FDA using the following nuclear parameters: $r_{0}=1.12 \mathrm{fm}$ and $\sigma=1.2 \mathrm{MeV} / \mathrm{fm}^{2}$. The static incompressibility $K$ for the finite nuclei is $A$-dependent. It was determined from a fit of the evaluated ISGMR energy to the experimental one $E_{0^{+}, \exp }$ of the giant-monopole resonance.

In the frequent-collision regime (a small $\beta$ in eq. (6)), the contribution to the sound velocity $c_{0}$ from the Fermi surface distortion effect is washed out and both energies $E 0$ and $E 1$ reach the first sound limit, i.e., $(E 1 / E 0)_{\mathrm{FDA}} \rightarrow$

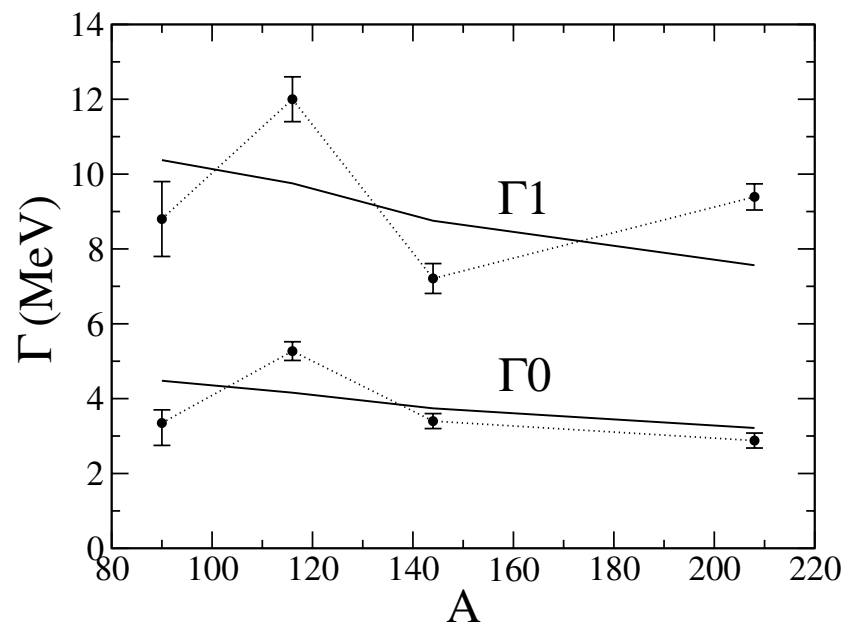

Fig. 2: Dependence of the ISGMR width, $\Gamma 0$, and the ISGDR width, $\Gamma 1$, on the mass number $A$. The FDA result is obtained using the relaxation time of eq. (6) with $\beta=4.6 \mathrm{MeV}$ and $\xi=0.59$ for $L=0$ and $\xi=0.21$ for $L=1$. The experimental data is from refs. [4,5].

$(E 1 / E 0)_{\mathrm{LDM}}=1.43$. The ratio $(E 1 / E 0)_{\mathrm{FDA}}$ grows with an increase of the relaxation time $\beta$ and approaches the zero sound limit for $\beta$. The transition from the zero to the first sound regime can be seen transparently in fig. 1 where the ratio $E 1 / E 0$ goes to the LDM limit at $\beta \rightarrow 0$ for several spherical nuclei: ${ }^{90} \mathrm{Zr},{ }^{116} \mathrm{Sn},{ }^{144} \mathrm{Sm}$ and ${ }^{208} \mathrm{~Pb}$.

The enhancement of the ratio $(E 1 / E 0)_{\mathrm{FDA}}$ with respect to the LDM estimate is due to the fact that the Fermi surface distortion effect on the energy $E 0$ of the lowest monopole mode is relatively small and $E 0$ appears close to the prediction of the classical LDM. Since the ISGDR appears as the overtone to the spurious mode, the Fermi surface distortion effect on the energy $E 1$ is much stronger and $E 1$ is shifted to higher energies with respect to the LDM prediction.

According to eqs. (9), (10) and (14), the position, $\hbar \omega_{R}$, and the width, $\Gamma=\hbar \gamma q^{2}$, of the compression mode depend on the relaxation time $\tau_{\text {eff }}$. In fig. 2 we have plotted the $A$-dependence of the width $\Gamma=\hbar \gamma q^{2}$ of the ISGMR, $\Gamma 0$, and the ISGDR, $\Gamma 1$, as evaluated for the two-body relaxation parameter $\beta=4.6 \mathrm{MeV}$. Note that this value of $\beta$ is consistent with those deduced in other works $[32,33]$. In eq. (7), the one-body relaxation parameter $\xi$ was derived from a fit of widths to the experimental data.

In fig. 3 we show the dependence of the energy ratio $E 1 / E 0$ on the nuclear mass number $A$. Considering the dependence of the FDA ratio $(E 1 / E 0)_{\mathrm{FDA}}$ on the relaxation time $\tau_{\text {eff }}$, we find a good agreement between experimental data and the results of the FDA model calculations (solid line 2) for the value of $\xi$ fitted to the widths $\Gamma 0$ and $\Gamma 1$ from fig. 2 . We wish to emphasize that it is conceptually important to achieve a description (on average) of both the energy ratio $E 1 / E 0$ (fig. 3 ) and the widths $\Gamma 0$ and $\Gamma 1$ (fig. 2) simultaneously, i.e., by using a common value for the relaxation parameters. 


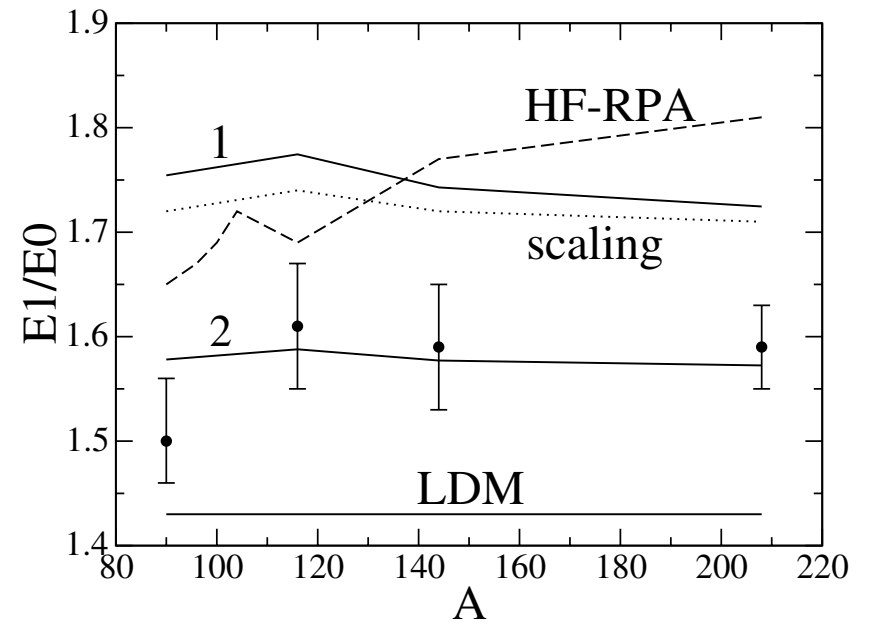

Fig. 3: Dependence of the energy ratio $E 1 / E 0$ on the nuclear mass number $A$. The ratio $(E 1 / E 0)_{\mathrm{FDA}}$ is obtained within the present FDA model with the relaxation parameter (see eq. (6)) $\beta \rightarrow \infty$ (solid line 1$)$. The solid line 2 is the FDA calculation with $\beta$ and $\xi$ from fig. 2 . The ratio $(E 1 / E 0)_{\mathrm{RPA}}$ (dashed line) is from fully self-consistent HF-RPA calculations [31]. The dotted line is the ratio $(E 1 / E 0)_{\text {scaling }}$ for the scaling model from eq. (25). The experimental ratio $(E 1 / E 0)_{\exp }$ is from refs. [4,5].

In fig. 3 the ratio $(E 1 / E 0)_{\mathrm{FDA}}$ for the rare-collision regime (solid line 1 ) was obtained using eqs. (13) and (16) for the limit $\tau_{\text {eff }} \rightarrow \infty$, and $(E 1 / E 0)_{\mathrm{RPA}}$ is obtained from the quantum HF-RPA calculations [31]. The ratio $(E 1 / E 0)_{\text {scaling }}$ (dotted line in fig. 3$)$ is obtained from the scaling model of ref. [7], where

$$
\begin{gathered}
(E 0)_{\text {scaling }}=\hbar \sqrt{\frac{K}{m\left\langle r^{2}\right\rangle}}, \\
(E 1)_{\text {scaling }}=\hbar \sqrt{\frac{7}{3} \frac{K+(27 / 25) \epsilon_{F}}{m\left\langle r^{2}\right\rangle} .}
\end{gathered}
$$

The ratios $(E 1 / E 0)_{\mathrm{RPA}}, \quad(E 1 / E 0)_{\text {scaling }}$ and $(E 1 / E 0)_{\mathrm{FDA}}$ in a rare-collision regime significantly exceed the LDM estimate $(E 1 / E 0)_{\mathrm{LDM}}$ and the experimental data $(E 1 / E 0)_{\exp }=1.6 \pm 0.1$ of refs. $[4,5]$. Note that both ratios $(E 1 / E 0)_{\mathrm{FDA}}$ and $(E 1 / E 0)_{\text {scaling }}$ have different asymptotic limits at $K \rightarrow \infty$. Namely, it can be seen from eq. (9) that $(E 1 / E 0)_{\mathrm{FDA}} \approx 1.43$ if $K \rightarrow \infty$, i.e., the Fermi-liquid drop ratio $(E 1 / E 0)_{\mathrm{FDA}}$ goes to the liquid-drop model limit $(E 1 / E 0)_{\text {LDM }}$ at $K \rightarrow \infty$, see also ref. [12]. This fact is important from the point of view of a consistent description of the compression modes within the Fermi-liquid theory. It is well known, see [13], that the zero sound velocity goes to the first sound limit at $K \rightarrow \infty$ and both energies $E 0$ and $E 1$ have to approach the corresponding LDM predictions. In this respect, the scaling model is incorrect because it predicts, see eq. (25), $(E 1 / E 0)_{\text {scaling }}=\sqrt{7 / 3}$ at $K \rightarrow \infty$.

It has been shown earlier (see, for example, [16]) that FDA approach is quite successful in describing the widths of giant resonances. We add that this approach allows us to assess the escape widths (particle decay widths) of giant resonances as a result of the particle splash effect [34]. Within the microscopic HF-based RPA approach, the escape widths can be assessed by carrying out continuumRPA calculations [30]. For heavy nuclei, the values of the escape widths are found to be about $10 \%$ of the total widths, in agreement with experiment $[25,26]$. Since the values of the escape widths are comparable to the current experimental uncertainties in the values of the total widths, we neglect the effect of the escape widths in the present work.

Our FDA calculations exhibit a non-monotonic dependence of the widths $\Gamma 0$ and $\Gamma 1$ on the relaxation time, which is a consequence of the memory effect ( $\omega$-dependence) in the friction coefficient $\gamma$, see eqs. (9) and (10). In the rare-collision regime the widths exhibit the quantum behavior, $\Gamma \sim 1 / \tau_{\text {eff }}$, while in the frequentcollision regime they have the hydrodynamic behavior, $\Gamma \sim \tau_{\text {eff. }}$ An essential point is that both energies $E 0$ and $E 1$ are monotonic functions of $\tau_{\text {eff }}$. Moreover, the ISGDR energy $E 1$ varies with $\tau_{\text {eff }}$ much faster than the ISGMR energy $E 0$, and $\Gamma 1$ is significantly larger than $\Gamma 0$.

We have carried out calculations of the centroid energies of the ISGMR and the ISGDR within the microscopic HFbased RPA and the semi-classical Fermi-liquid approach. We have adopted a Fermi-liquid model with two essential features: i) the linearized kinetic equation is applied to the nuclear interior, where the relatively small oscillations of the particle density take place; ii) the dynamics in the surface layer of the nucleus is described by means of the macroscopic boundary condition which is taken as a condition for the balance of the forces on the free nuclear surface. We demonstrated that one can obtain an agreement with the experimental data on $E 1 / E 0$ in the presence of relaxation processes. Besides the collisional width, the experimentally observable widths of the ISGMR and the ISGDR include the fragmentation width. Within our semi-classical kinetic theory, this mechanism of resonance spreading is considered an additional relaxation effect (one-body relaxation) due to the single-particle scattering on the moving surface of the nucleus. Finally, we would like to note that the semi-classical kinetic approach, used in this article, is highly convenient for the study of the averaged properties of the nuclear dynamics. This approach provides information on the macroscopic characteristics without detailed knowledge of the wave function of the nucleus. Here the claim is to describe the general features of collective excitations, such as the $A$-dependence of the widths $\Gamma 0$ and $\Gamma 1$ and the ratio $E 1 / E 0$ in a systematic way, ignoring many quantum effects, e.g., the shell structure effects.

$* * *$

This work was supported in part by the US Department of Energy under grant No. DOE-FG03-93ER40773. 


\section{REFERENCES}

[1] Morsch H. P., Rogge M., Turek P. and MayerBoricke C., Phys. Rev. Lett., 45 (1980) 337.

[2] Davis B. et al., Phys. Rev. Lett., 79 (1997) 609.

[3] Clark H. L., Lui Y.-W., Youngblood D. H., Bachtr K., Garg U., HarakeH M. N. and KalantarNayestanski N., Nucl. Phys. A, 649 (1999) 57.

[4] Youngblood D. H., Clark H. L. and Lui Y.-W., Phys. Rev. C, 69 (2004) 034315.

[5] Youngblood D. H., Clark H. L. and Lui Y.-W., Phys. Rev. C, 69 (2004) 054312.

[6] Deal T. J., Nucl. Phys. A, 217 (1973) 210.

[7] Stringari S., Phys. Lett. B, 108 (1982) 232.

[8] Van Giai N. and Sagawa H., Nucl. Phys. A, 371 (1981) 1.

[9] Harakeh M. N. and Diepernik A. E. L., Phys. Rev. C, 23 (1981) 2329.

[10] Hamamoto I., Sagawa H. and Zhang X. Z., Phys. Rev. C, 57 (1998) R1064.

[11] Kolomiets A., Kolomietz V. M. and Shlomo S., Phys. Rev. C, 59 (1999) 3139.

[12] Kolomietz V. M. and Shlomo S., Phys. Rev. C, 61 (2000) 064302.

[13] Abrikosov A. A. and Khalatnikov I. M., Rep. Prog. Phys., 22 (1959) 329.

[14] Lifshitz E. M. and Pitajevskin L., Physical Kinetics (Nauka, Moscow) 1978.

[15] Baym G. and Pethick C., Landau Fermi-Liquid Theory (John Wiley and Sons, New York) 1991.

[16] Kolomietz V. M. and Shlomo S., Phys. Rep., 390 (2004) 133.

[17] Kolomietz V. M. and Tang H. H. K., Phys. Scr., 24 (1981) 915.
[18] Brooker G. A. and Sykes J., Ann. Phys. (N.Y.), 61 (1970) 387.

[19] Kolomietz V. M., Plujko V. A. and Shlomo S., Phys. Rev. C, 54 (1996) 3014.

[20] Di Toro M., Kolomietz V. M. and Larionov A. B., Phys. Rev. C, 59 (1999) 3099.

[21] Griffin J. J. and Dworzecka M., Phys. Lett. B, 156 (1985) 139.

[22] Yannouleas C., Nucl. Phys. A, 439 (1985) 336.

[23] Yannouleas C. and Broglia R. A., Ann. Phys. (N.Y.), 217 (1992) 105.

[24] Sierk A. J., Koonin S. E. and Nix J. R., Phys. Rev. C, 17 (1978) 646.

[25] Borghols W. T. A. et al., Nucl. Phys. A, 515 (1990) 173.

[26] Brandenburg S. et al., Nucl. Phys. A, 466 (1987) 29; Phys. Rev. C, 39 (1989) 2448.

[27] Shlomo S., Kolomietz V. M. and Agrawal B. K., Phys. Rev. C, 68 (2003) 064301.

[28] Kolomietz V. M., Magner A. G., Strutinsky V. M. and Vydrug-Vlasenko S. M., Nucl. Phys. A, $\mathbf{5 7 1}$ (1994) 117.

[29] Bohr A. and Mottelson B., Nuclear Structure, Vol. 2 (Benjamin, New York) 1975.

[30] Shlomo S. and Bertsch G. F., Nucl. Phys. A, 243 (1975) 507.

[31] Tapas Sil, Shlomo S., Agrawal B. K. and Reinhard P.-G., Phys. Rev. C, 73 (2006) 034316.

[32] Auerbach N. and Yeverechyahu A., Ann. Phys. (N.Y.), 95 (1975) 35.

[33] Auerbach N. and Shlomo S., Phys. Rev. Lett., 103 (2009) 172501.

[34] Kolomietz V. M., Nucl. Phys. A, 743 (2004) 211. 